\title{
Dynamical diffraction theory of waves in photonic crystals built from anisotropically scattering elements
}

\author{
V. Baryshevsky, E. Gurnevich \\ Research Institute for Nuclear Problems BSU, Belarus
}

The process of refraction and diffraction of waves in natural and artificial crystals built from anisotropically scattering elements is considered. As an example, the refraction of electromagnetic waves in two-dimensional photonic crystal formed from parallel metallic threads is studied in details. The expression for the effective scattering amplitude of the wave by a thread (in the crystal) is found for the case when the scattering by a single thread is anisotropic. It is shown that, unlike the case of a wave with polarization parallel to the threads, the refractive index for waves with orthogonal polarization can be greater than 1 ; this leads to the possibility of Cherenkov radiation in such a crystal. A system of equations describing the dynamical diffraction of waves in photonic crystals is obtained. The properties of parametric ("quasi-Cherenkov") radiation of charged particles in photonic crystals are considered. Since to describe the process of the scattering the most general approach is used, the obtained results are valid for a wide range of problems, not limited by considering only electromagnetic waves, or only specific type of crystals. In particular, they may be useful when studying diffraction of cold neutrons in artificial crystals.

NOTES

\section{Plasmon-enhanced luminescence of fluorescein-labeled biomolecules on top of silver nanostructures}

\author{
A. Ramanenka ${ }^{1}$, S. Vaschenko ${ }^{1}$, D. Guzatov ${ }^{2}$, S. Gaponenko ${ }^{3}$ \\ ${ }^{1}$ Belarusian State University, Belarus; \\ ${ }^{2}$ Yanka Kupala Grodno State University, Belarus; \\ ${ }^{3}$ Stepanov Institute of Physics, NAS Belarus, Belarus
}

Plasmonic enhancement of luminescence in nanostructures has big application potential but many proposed nanostructures are expensive and based on complicated procedures. The real-life implementation needs cheap, easy to synthesis, durable and reproducible plasmonic nanotechnology. We proposed a simple method of plasmonic nanostructures formation using silver colloidal films and polyelectrolyte layers as a dielectric spacer. Bovine serum albumin molecules labeled by fluorescein isothiocyanate were used as model biological targets. Silver sols with different nanoparticle sizes were synthesized by AgNO3 reduction using sodium citrate. To develop a dielectric spacer between a silver film and biomolecules, the substrate surface was covered by alternate polyelectrolyte layers, followed by aqueous solution of labeled molecules. Luminescence excitation was performed by LED $(460 \mathrm{~nm})$. Plasmonic effects on biomolecules luminescence for different silver nanoparticle sizes, excitation light polarization, and molecule-silver spacing were examined. The enhancement factor exhibited smooth dependence upon polyelectrolyte spacer thickness with maximum for 5 layers. For unpolarized excitation the highest enhancement factor was 7 for $50-90 \mathrm{~nm}$ silver nanoparticles. The maximal 9-fold enhancement was observed for p-polarized excitation. Experimental results are in good agreement with performed theoretical calculations taking into account local intensity enhancement, radiative and nonradiative rates modification, light polarization, molecule position and its dipole moment orientation.

NOTES 